\title{
PRMT7 contributes to the metastasis phenotype in human non-small-cell lung cancer cells possibly through the interaction with HSPA5 and EEF2
}

This article was published in the following Dove Press journal:

OncoTargets and Therapy

\author{
Dezhi Cheng ${ }^{1,2}$ \\ Zhifeng $\mathrm{He}^{2}$ \\ Liangcheng Zheng ${ }^{2}$ \\ Deyao $\mathrm{Xie}^{2}$ \\ Shangwen Dong' \\ Peng Zhang' \\ 'Department of Thoracic Surgery, \\ Tianjin Medical University General \\ Hospital, Tianjin, China; ${ }^{2}$ Department \\ of Thoracic Cardiovascular, The \\ First Affiliated Hospital of Wenzhou \\ Medical University, Wenzhou, China
}

Correspondence: Peng Zhang Department of Thoracic Surgery, Tianjin Medical University General Hospital, I54 Anshan Road, Heping District,

Tianjin City, China

Tel +8602260362636

Fax +8602260362636

Email wings_spread@I26.com
Background: Non-small-cell lung cancer (NSCLC) constitutes the leading cause of cancer death in humans. Previous studies revealed the essential role of the protein arginine methyltransferase 7 (PRMT7) in promoting metastasis in breast cancer. However, its function and potential mechanism in NSCLC remain unclear.

Materials and methods: The gene expression of PRMT7 between lung cancer tissues and normal tissues was studied with online database (http://medicalgenome.kribb.re.kr/ GENT/). NSCLC cell lines with specific gene overexpression were constructed with lentivirus transduction. Matrigel invasion and colony formation assays were performed to evaluate the invasion and colony formation abilities. Co-immunoprecipitation coupled with mass spectrometry analysis was performed to explore the potential interaction proteins of PRMT7. Bioinformatic analysis was performed with Gene Ontology and Kyoto Encyclopedia of Genes and Genomes databases.

Results: Online analysis of gene expression patterns revealed the relatively high expression of PRMT7 in lung cancer tissues. PRMT7 overexpression was able to promote the invasion and colony formation of A549 and SPC-A1 cells. A total of 19 in-common proteins shared by both NSCLC cell lines were identified to be interacting with PRMT7 and found to participate in a wide variety of pathways and protein-protein interactions according to bioinformatic analysis. Among them, HSPA5 and EEF2 were further investigated for their essential roles in PRMT7promoted NSCLC cell invasion.

Conclusion: Our results suggested PRMT7 overexpression was able to promote metastasis in NSCLC possibly through the interaction with HSPA5 and EEF2, which provides the potential mechanism of oncogenesis in lung cancer.

Keywords: human non-small-cell lung cancer, arginine methylation, $P R M T 7$, protein-protein interaction, $H S P A 5, E E F 2$

\section{Introduction}

Lung cancer, characterized by a poor prognosis, is among the most deadly cancers all over the world, ${ }^{1}$ which can be divided into two main subtypes, small-cell lung carcinoma and non-small-cell lung carcinoma (NSCLC). ${ }^{2}$ Of these, NSCLC accounts for the majority $(>80 \%$ ) of lung cancer cases. More importantly, NSCLC is often detected in the late stage of metastasis, which makes effective treatment impossible. ${ }^{3}$ In a previous report, it was suggested that metastasis in lung cancer is a multifaceted process, which still remains unclear in respect to the molecular basis of mechanism. ${ }^{4}$ Together, these represent great challenges in the study of NSCLC as well as in its clinical management. ${ }^{5}$ 
Arginine methylation, a common posttranslational modification, ${ }^{6}$ includes three types of methylarginine products in mammalian cells, for example, $\omega-N^{G}$-monomethylarginine, $\omega$ - $N^{G}, N^{G}$-asymmetric dimethylarginine, and $\omega-N^{G}, N^{\prime}{ }_{-}$ symmetric dimethylarginine. ${ }^{7}$ The formation of methylarginine is catalyzed by the family of protein arginine methyltransferases (PRMTs), which consist of nine different members and can be divided into three different types (types I, II, and III). ${ }^{7,8}$ Accumulating studies have indicated the deregulation of PRMTs is implicated in the pathogenesis of cancers. ${ }^{7,9-11}$

PRMT7 is a unique member characterized by both type II and type III enzyme activities. ${ }^{7}$ PRMT7 has been shown to be extensively involved in a wide variety of biological processes, ${ }^{12}$ such as DNA transcription, RNA splicing, DNA damage repair, metastasis, and pluripotency. ${ }^{13-15}$ Moreover, PRMT7 is required for the snRNP assembly, which is a major component of the spliceosome. ${ }^{16}$ Recently, PRMT7 was recognized as a metastasis promoter gene in breast cancer, which could be attributed to the induction of epithelial-tomesenchymal transition by PRMT7. ${ }^{17,18}$ Furthermore, Jelinic et al demonstrated PRMT7 was able to interact with the proposed oncogene CCCTC binding factor-like (CTCFL), ${ }^{19}$ which increased its methyltransferase activity. ${ }^{20} \mathrm{PRMT7}$ also showed promise in the anticancer therapies, which could be supported by the fact that PRMT7 downregulation sensitized Hela cells to the inhibitor of DNA topoisomerase I, camptothecin. ${ }^{21}$

However, little is known about the function of PRMT7 in NSCLC, despite the knowledge on its participation in numerous essential processes. To investigate its potential role, we constructed the PRMT7-overexpressed NSCLC cell models and evaluated its effects on cancer metastasis. We also explored the interacting proteins of PRMT7, among which HSPA5 and EEF2 were highlighted owing to the established contributions to the invasion of NSCLC cells. Our findings laid the basis for further study of the molecular basis of mechanism, which could help in the development of targeted chemotherapy against NSCLC.

\section{Materials and methods Cell lines}

Human lung adenocarcinoma A549 and SPC-A1 cells were purchased from the Institute of Biochemistry and Cell Biology, Chinese Academy of Sciences (Shanghai, China). HEK293T cells were purchased from ATCC (No. CRL-11268; American Type Culture Collection, Manassas, VA, USA). Cells were grown in DMEM medium (Thermo Fisher Scientific, Waltham, MA, USA) supplemented with
$10 \%$ heat-inactivated fetal bovine serum (FBS; Thermo Fisher Scientific), $100 \mathrm{U} \mathrm{mL}^{-1}$ penicillin, and $100 \mu \mathrm{g} \mathrm{mL}^{-1}$ streptomycin (Mediatech Inc., Manassas, VA, USA) in a humidified atmosphere of $5 \% \mathrm{CO}_{2}$ at $37^{\circ} \mathrm{C}$.

\section{PRMT7 expression in lung cancer tissues}

The analysis of gene expression of PRMT7 in both normal and cancer lung tissues was performed with the online database Gene Expression across Normal and Tumor tissue (http://medicalgenome.kribb.re.kr/GENT/). ${ }^{22}$

\section{Vector construction}

RPMT7 overexpression vector was constructed by cloning the cDNAs of human RPMT7 into a PSE2130 vector. The constructs were confirmed by sequencing.

\section{Gene overexpression and knockdown}

pSE2130 retroviral vectors were used to study gene overexpression of RPMT7. EEF2 and HSPA 5 were knocked down with a pLVT1568-based lentiviral vector. Lentiviruses were generated in HEK293T cells as previously described. ${ }^{23}$ A549 and SPC-A1 cells were infected with virus-containing supernatants. Alterations in the expression of RPMT7, EEF2, and HSPA5 were confirmed by Western blotting or quantitative reverse transcriptase-polymerase chain reaction.

\section{RNA isolation and quantitative reverse transcriptase-polymerase chain reaction}

Total RNA was extracted from the cultured cells with TRIzol reagent according to the standard manual. RNA was reverse transcribed using Revert Aid First Strand cDNA Synthesis Kit before quantification with spectrophotometry. Maxima SYBR Green/ROX qPCR Master Mix was used for real-time polymerase chain reaction experiment. GAPDH was used as an internal control.

\section{Cell invasion assay}

Cells' invasion ability was evaluated as described previously. ${ }^{24}$ Briefly, cells $\left(1 \times 10^{5}\right)$ cultured in $100 \mu \mathrm{L}$ FBSfree medium were seeded into transwell chambers which were precoated with Matrigel. Medium containing 20\% FBS was added to the lower chamber. After culturing for $72 \mathrm{~h}$ at $37^{\circ} \mathrm{C}$, non-invading cells were removed with cotton swabs. Invading cells were fixed with $4 \%$ para-formaldehyde and stained with crystal violet solution. Data were quantified by ImageJ.

\section{Colony formation assay}

Colony formation assays were performed as described before. ${ }^{25}$ Briefly, $2 \mathrm{~mL}$ of 250 cells per well were seeded into 
a six-well plate. After culturing in the cell incubator $\left(37^{\circ} \mathrm{C}\right.$, $5 \% \mathrm{CO}_{2}$ ) for 10 days, the colonies per well were fixed with ethanol, stained with crystal violet solution, and observed with a microscope. Data were quantified by ImageJ.

\section{Immunoprecipitation and liquid chromatography with tandem mass spectrometry protein identification}

Immunoprecipitation was carried out as previously reported. ${ }^{26}$ Cells overexpressing RPMT7 were harvested and lysed with immunoprecipitation (IP) lysis buffer (Biyuntian, Shanghai, China). After centrifugation at $16,000 \times g$ at $4^{\circ} \mathrm{C}$ for 15 minutes, the supernatants were collected and the protein concentration was quantified with bicinchoninic acid reagent. Then, the samples were pre-cleared with protein $\mathrm{A} / \mathrm{G}$ magnetic beads (Thermo Fisher Scientific). After that, $500 \mu \mathrm{g}$ of total protein was incubated with $5 \mu \mathrm{g}$ of antibody or IgG at $4^{\circ} \mathrm{C}$ overnight. Then, $25 \mu \mathrm{L}$ protein $\mathrm{A} / \mathrm{G}$ magnetic beads was added and incubated at $4^{\circ} \mathrm{C}$ for another 1 hour. After washing, the proteins were eluted with sodium dodecyl sulfate sample buffer and separated with sodium dodecyl sulfate-polyacrylamide gel electrophoresis. The gel lanes were excised, destained, washed, dried, and dehydrated with acetonitrile. Finally, the peptides were extracted, concentrated, and measured with liquid chromatography with tandem mass spectrometry.

\section{Results}

\section{PRMT7 expression is higher in lung cancer tissues than in normal tissues}

Previously, gene expression meta-analysis identified PRMT7 as a candidate gene involved in breast cancer metastasis. ${ }^{17}$ Later, Baldwin et al reported the overexpression of PRMT7 promotes cell invasion in breast cancer. ${ }^{27}$ Similarly, Yao et al showed PRMT7 is expressed at a higher level and mediates the induction of epithelial-to-mesenchymal transition and metastasis in breast carcinoma cells. ${ }^{18}$ These studies consistently indicate $P R M T 7$ as a promising biomarker and therapeutic target for breast cancer. However, not much has been reported on the function of PRMT7 in the metastasis of NSCLC, which constitutes the primary goal of the current work.

To gain insights into the potential role of PRMT7 in NSCLC, we analyzed the public data from the online database (Gene Expression across Normal and Tumor tissue), which provides the expression patterns of a specific gene, for example, PRMT7, across both normal and cancer tissues of humans on the basis of profiling with Affymetrix U133plus2 platform. It helps to facilitate the identification of cancer outliers overexpressed only in a subset of patients. ${ }^{22}$

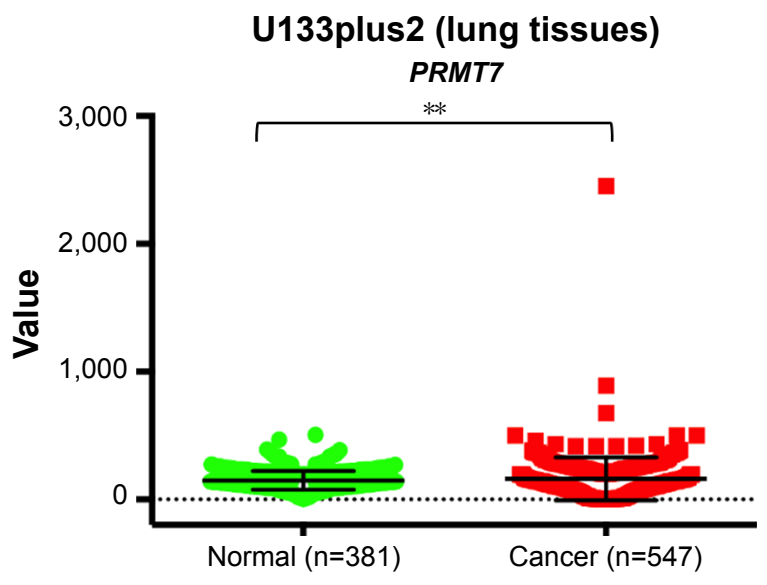

Figure I Expression patterns of PRMT7 in normal and cancerous lung tissues from analysis of UI33plus2 dataset (http://medicalgenome.kribb.re.kr/GENT/).

Note: PRMT7 is significantly upregulated in lung cancer tissues compared to normal controls $(* * P<0.01)$.

Our results showed that the expression of PRMT7 was significantly higher in lung cancer tissues than in normal tissues (Figure 1), suggesting the potential role of PRMT7 in the oncogenesis of lung cancer.

\section{PRMT7 overexpression promotes the invasion and colony formation of NSCLC cells}

To investigate the potential roles of PRMT7 in NSCLC, we constructed PRMT7-overexpressed A549 and SPC-A1 cell models with lentiviral transduction method. ${ }^{28}$ The expression levels of PRMT7 were assessed with Western blot analysis in both cell lines, which confirmed significantly upregulated expression of PRMT7 (Figure 2A).

On this basis, we evaluated the effects of PRMT7 overexpression on the metastasis phenotype of NSCLC cells. In the Matrigel invasion experiment, our results showed that PRMT7 overexpression significantly increased the cell invasion of both A549 and SPC-A1 cells compared to the vector controls (Figure 2B), which can be evidenced by the enhanced intensity of crystal violet staining. Consistently, the quantitative results confirmed the above observations, with statistical significance found in the differences $(P<0.05)$, as shown in Figure 2C.

We also performed the colony formation assays and found the overexpression of $P R M T 7$ largely promoted the colonyforming ability in both A549 and SPC-A1 cells compared to the vector controls (Figure 2D), which was further confirmed by the quantification and statistical analysis $(P<0.05)$, as shown in Figure 2E.

Together, these results clearly indicated $P R M T 7$ overexpression was able to contribute to the metastasis phenotype of NSCLC cells, confirming the essential role of PRMT7 in the 
A

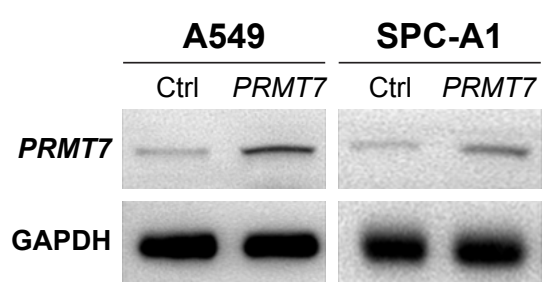

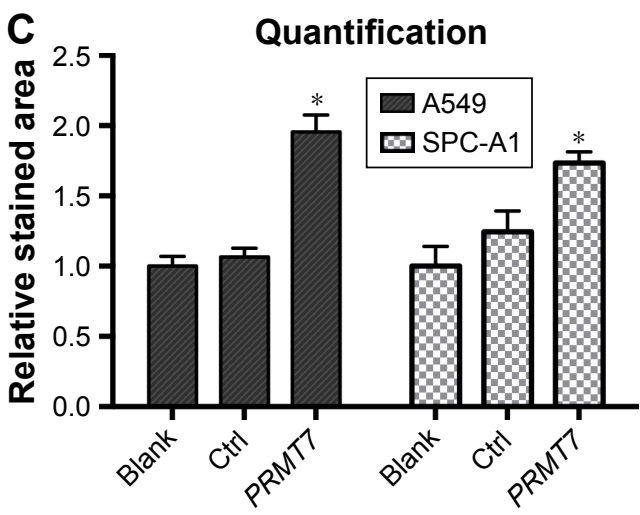

Ctrl

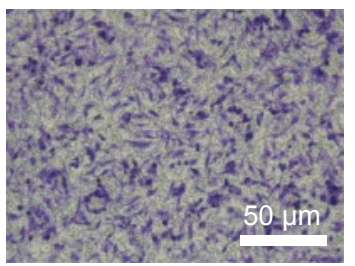

PRMT7
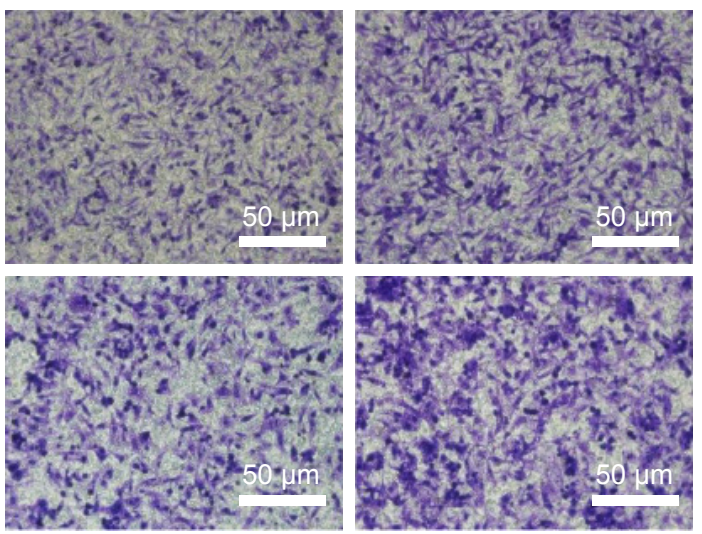

B

Blank
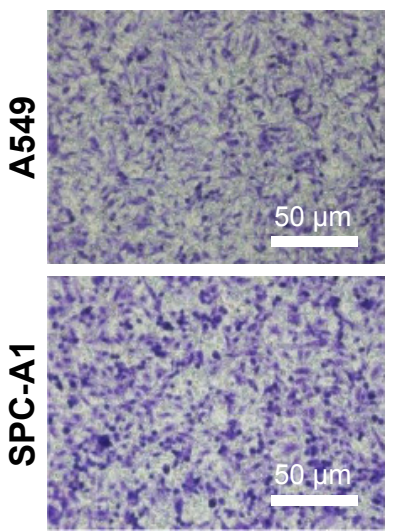

D Blank

ํำ
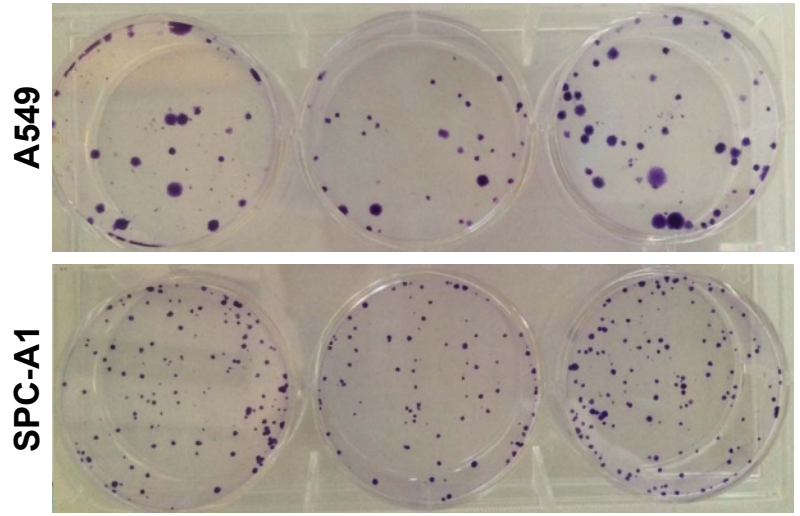

E

Cell colony count

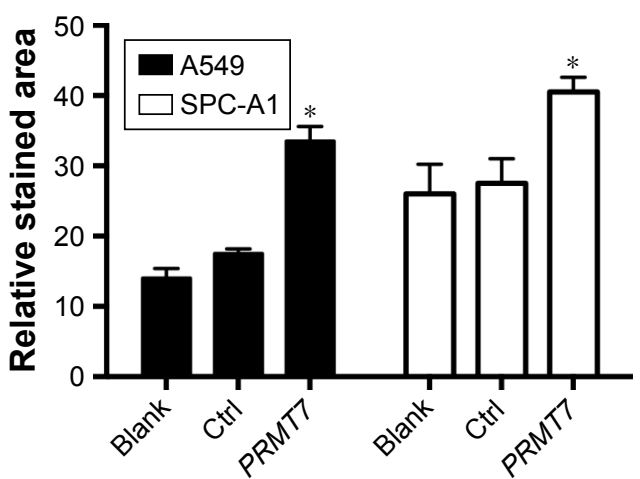

Figure 2 Overexpression of PRMT7 in A549 and SPC-AI cells promoted cellular invasion and colony formation.

Notes: (A) Expression level of PRMT7 in A549 and SPC-AI cells was measured with Western blot analysis. (B) Invasion of A549 and SPC-AI cells was enhanced by PRMT7 overexpression. Images were collected from a representative experiment. (C) Quantitative results of cell invasion. (D) Colony formation of A549 and SPC-AI cells was promoted by PRMT7 overexpression. Images were collected from a representative experiment. (E) Quantitative results of cell colony formation. $* P<0.05$, compared to control group.

Abbreviation: Ctrl, control.

oncogenesis of NSCLC. Our results are in agreement with previous findings that PRMT could promote oncogenesis in cancer. $^{27}$

\section{Exploring PRMT7-interacting proteins in both A549 and SPC-AI cells}

On the above basis, we can see PRMT7 contributes to the metastasis phenotype of NSCLC cells. However, little is known about the molecular basis of the mechanism. Recently, Jain et al reported the epigenetic control via allosteric regulation of PRMTs ${ }^{29}$ which inspired us to investigate the interacting proteins for PRMT7. To this end, we performed co-immunoprecipitation experiments against PRMT7 in both A549 and SPC-A1 cells, which were followed by silver staining and mass spectrometry analysis. As a result, a collection of 79 candidate proteins were recognized, from which 
Table I Nineteen in-common proteins shared by both A549 and SPC-AI cells, which were identified

\begin{tabular}{|c|c|c|c|c|}
\hline No. & Protein full name & $\begin{array}{l}\text { Protein } \\
\text { short name }\end{array}$ & Score & $\begin{array}{l}\text { Mass } \\
\text { (Dalton) }\end{array}$ \\
\hline I & Desmoplakin & DSP & 43 & 334,021 \\
\hline 2 & Alpha-actinin-4 & ACTN4 & 648 & 105,245 \\
\hline 3 & Neutral alpha-glucosidase $A B$ & GANAB & 175 & 96,441 \\
\hline 4 & Elongation factor 2 & EEF2 & 166 & 96,246 \\
\hline 5 & Desmocollin-I & $\mathrm{DSCl}$ & 62 & 101,406 \\
\hline 6 & 78 kDa glucose-regulated protein & HSPA5 & 4,301 & 72,402 \\
\hline 7 & Procollagen galactosyltransferase I & COLGALTI & 554 & 71,933 \\
\hline 8 & Endoplasmin & HSP90BI & 365 & 92,696 \\
\hline 9 & $\begin{array}{l}\text { Procollagen-lysine,2-oxoglutarate } \\
\text { 5-dioxygenase } 3\end{array}$ & PLOD3 & 138 & 85,302 \\
\hline 10 & $\begin{array}{l}\text { ATP-dependent RNA helicase } \\
\text { DDX3X }\end{array}$ & DDX3X & 134 & 73,597 \\
\hline II & Moesin & MSN & 126 & 67,892 \\
\hline 12 & Polyadenylate-binding protein I & PABPCI & 96 & 70,854 \\
\hline 13 & Protein disulfide-isomerase A4 & PDIA4 & 49 & 73,229 \\
\hline 14 & $\begin{array}{l}\text { Dihydrolipoyllysine-residue } \\
\text { acetyltransferase component of } \\
\text { pyruvate dehydrogenase complex }\end{array}$ & DLAT & 2,197 & 69,466 \\
\hline 15 & $\begin{array}{l}\text { X-ray repair cross-complementing } \\
\text { protein } 6\end{array}$ & $\mathrm{XRCC6}$ & 932 & 70,084 \\
\hline 16 & Prelamin-A/C & LMNA & 260 & 74,380 \\
\hline 17 & Stress-70 protein, mitochondrial & HSPA9 & 235 & 73,920 \\
\hline 18 & $\begin{array}{l}\text { Sodium/potassium-transporting } \\
\text { ATPase subunit alpha-2 (fragment) }\end{array}$ & ATPIA2 & 138 & 79,827 \\
\hline 19 & $\begin{array}{l}\text { ATP synthase subunit beta } \\
\text { (fragment) }\end{array}$ & ATP5B & 50 & 38,226 \\
\hline
\end{tabular}

19 in-common targets shared by both A549 and SPC-A1 cell lines were selected for further studies (Table 1).

\section{Gene Ontology, Kyoto Encyclopedia of Genes and Genomes pathway, protein binding analysis of the identified proteins}

Gene Ontology (GO) annotation was performed on 19 incommon proteins shared by both A549 and SPC-A1 cells. The top eight categories are shown in Table 2. Notably, the term of cell motility got the highest rank, which was followed by acetyl-CoA biosynthesis, proton/hydrogen transport, non-recombinational repair, and double-strand break repair. Given the experimentally established evidences that tumor cell motility is essential for the progression of cancer metastasis, ${ }^{30}$ our results provide insights into the molecular basis underlying the PRMT7-promoted metastasis phenotype of NSCLC cells.

We also performed Kyoto Encyclopedia of Genes and Genomes (KEGG) pathway and binding protein analysis on the above 19 target proteins. Our results showed that the proteins were primarily involved in prion disease, regulation of actin cytoskeleton, ATP synthesis, pyruvate metabolism, and MAPK signaling pathway (Table 3). Moreover, the 19 PRMT7-interacting proteins are likely to bind with APOB, NCF4, PKP2, PXN, PSME3, PECAM1, JUP, and PAIP1 (Table 4). In particular, heat shock $70 \mathrm{kDa}$ protein 5 (HSPA5) was found to participate in a wide variety of pathways and protein-protein interactions, suggesting its complex role in regulating cell signaling and metabolism.

\section{Validation of identified PRMT7-interacting proteins}

To validate the above experimental results, we selected two candidates from 19 in-common proteins for further investigations, including HSPA5 and EEF2. Our selection was mainly based on the previous reports that both of them are involved in cancer metastasis. ${ }^{31,32}$ For example, Chen et al found the expression of HSPA5 was positively correlated with the metastatic events in triple-negative breast cancer patients. ${ }^{31}$ Moreover, downregulation of HSPA5 significantly abolished cancer metastasis in the xenograft model. ${ }^{31}$ Song et al revealed that the overexpression of EEF2 increased the cell migration and invasion in lung squamous cell carcinoma. ${ }^{32}$ On these bases, we were encouraged to investigate the effects of $H S P A 5$ and $E E F 2$ on the oncogenic function of PRMT7.

We performed standard co-immunoprecipitation experiment to precipitate $P R M T 7$ complex with anti-PRMT7 antibody in both A549 and SPC-A1 cells. Then, we detected the target proteins in the co-precipitated products with

Table 2 GO analysis of 19 in-common proteins shared by both A549 and SPC-AI cells

\begin{tabular}{llll}
\hline No. & GO terms & Genes & P-value \\
\hline$I$ & Cell motility & ACTN4, ATPIA2, MSN & 0.0008 \\
2 & Acetyl-CoA biosynthesis & DLAT & $0.00 I$ \\
3 & Proton transport & ATPIA2, ATP5B & 0.002 \\
4 & Hydrogen transport & ATPIA2, ATP5B & 0.002 \\
5 & ATP hydrolysis coupled proton transport & ATPIA2 & 0.003 \\
6 & Non-recombinational repair & G22PI & 0.003 \\
7 & Double-strand break repair via nonhomologous end joining & G22PI & 0.003 \\
8 & Energy-coupled proton transport against electrochemical gradient & ATPIA2 & 0.005 \\
\hline
\end{tabular}

Abbreviation: GO, Gene Ontology. 
Table 3 KEGG pathway analysis of 19 in-common proteins shared by both A549 and SPC-AI cells

\begin{tabular}{llll}
\hline No. & KEGG pathway & Genes & P-value \\
\hline I & Prion disease & HSPA5 & 0.004 \\
2 & Regulation of actin cytoskeleton & ACTN4, MSN & 0.01 \\
3 & ATP synthesis & ATP5B & 0.01 \\
4 & Pyruvate metabolism & DLAT & 0.01 \\
5 & MAPK signaling pathway & HSPA5, HSPA9B & 0.02 \\
\hline
\end{tabular}

Abbreviation: KEGG, Kyoto Encyclopedia of Genes and Genomes.

Western blot analysis. Our results showed that the HSPA5 and EEF2 bands were clearly present in the immunoblots of PRMT7 co-precipitants in both A549 and SPC-A1 cells (Figure 3). In contrast, only weak band of similar molecular weight was detected in the control precipitants (Figure 3). These results validated that PRMT7 interacts with HSPA5 and EEF2 directly in both NSCLC cell lines.

\section{HSPA5 and EEF2 mediated PRMT7- induced metastasis phenotype in NSCLC cells}

We further investigated the function of HSPA5 and EEF2 in mediating $P R M T 7$-induced metastasis in NSCLC. Based on the above results, it was found that PRMT7 overexpression was able to promote invasion in both A549 and SPC-A1 cell lines. We screened a collection of siRNAs by transfecting them into NSCLC cells to evaluate the knockdown effect against a specific gene (HSPA5 or EEF2). The relative mRNA expressions of both target genes are shown in Figure 4A. The siRNAs with significant effect and balanced performance between both cell lines were selected for the following experiment (HSPA5: si-2082 and EEF2: si-683).

Afterward, we transfected the selected siRNAs into PRMT7-overexpressed A549 and SPC-A1 cells and assessed their effects on the cell invasion with Matrigel invasion assays. From our results, it was found that compared to the vector control, knockdown of HSPA5 or EEF2 gene significantly reduced $P R M T 7$-promoted invasion of NSCLC cells, as evidenced by the decreased intensity of crystal violet staining in both A549 and SPC-A1 cells (Figure 4B). Quantitative results further confirmed our observations with a statistically significant difference $(P<0.05$; Figure $4 C)$. Collectively, these results suggested that HSPA5 and EEF2 may mediate $P R M T 7$-induced NSCLC cell invasion through directly interacting with $P R M T 7$.

\section{Discussion}

PRMTs participate in a wide variety of physiologic and pathogenetic processes. ${ }^{7,33,34}$ As the unique member characterized by both type II and III enzyme activity, PRMT7 was found to be a metastasis promoter gene in breast cancer. ${ }^{17,18,27}$ In the present work, we found PRMT7 expression was significantly increased in the lung cancer tissues by analyzing the target gene expression patterns in $>34,000$ human samples. Moreover, overexpression of PRMT7 in two different NSCLC cell lines (A549 and SPC-A1) was found to promote the cell invasion and colony formation consistently, suggesting PRMT7 plays an important role in promoting the metastasis phenotype of NSCLC cells.

A recent report published in 2017 revealed the new mechanism of epigenetic control via allosteric regulation of PRMTs. ${ }^{29}$ In order to explore the molecular basis underlying the contribution of PRMT7 to the cell invasion in NSCLC, we performed co-immunoprecipitation experiments and mass spectrometry to explore the potential interacting proteins for PRMT7. As a result, 79 candidates were identified and 19 in-common genes shared by both A549 and SPC-A1 cell lines were highlighted, which constitute the focus of following studies. To our knowledge, there is no report focusing on the PRMT7-interacting regulation proteins in NSCLC. Our results laid the basis for the investigation of mechanism as well as target-based drug discovery.

Bioinformatic analysis, for example, GO annotation, revealed the close relationship between the above identified proteins and cell motility, which is known to contribute to

Table 4 Binding protein analysis of 19 in-common proteins shared by both A549 and SPC-AI cells

\begin{tabular}{llll}
\hline No. & Binding proteins & Genes & P-value \\
\hline I & APOB: apolipoprotein B (including Ag(x) antigen) & ERP70, HSPA5, TRAI & $<0.000$ I \\
2 & NCF4: neutrophil cytosolic factor 4, 40 kDa & G22PI, MSN & $<0.000$ I \\
3 & PKP2: plakophilin 2 & DSCI, DSP & 0.0004 \\
4 & PXN: paxillin & G22PI, HSPA5, LMNA, PABPCI & 0.0006 \\
5 & PSME3: proteasome (prosome, macropain) activator subunit 3 & ATP5B, HSPA5 & 0.0006 \\
6 & PECAMI: platelet/endothelial cell adhesion molecule (CD3I) & DSP, G22PI & 0.002 \\
7 & JUP: junction plakoglobin & DSCI, DSP & 0.005 \\
8 & PAIPI: poly(A) binding protein interacting protein I & PABPCI & 0.005 \\
\hline
\end{tabular}




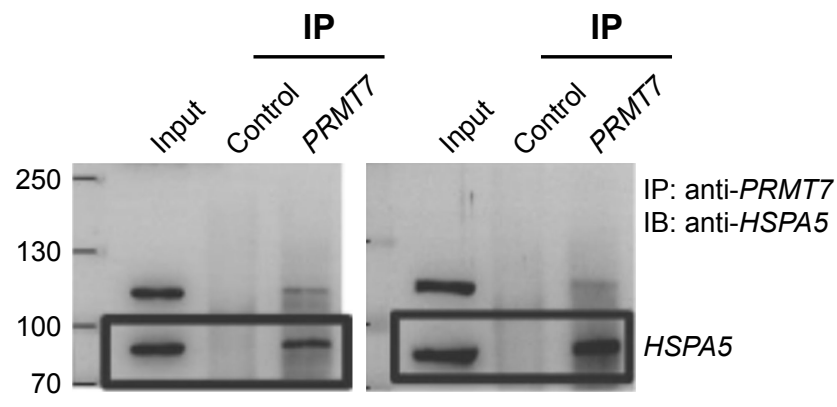

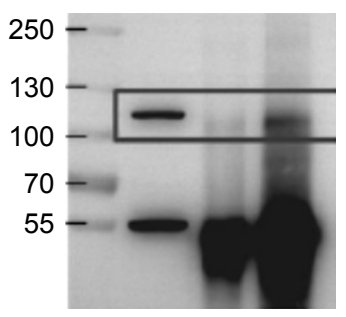

A549

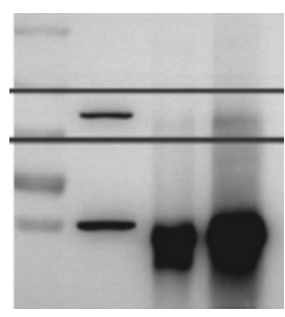

SPC-A1
Figure 3 Validation of the interactions between PRMT7 and HSPA5, EEF2 by co-immunoprecipitation and Western blot analysis in A549 and SPC-AI cells. Abbreviations: IP, immunoprecipitation; IB, immunoblot.

the progression of cancer metastasis. ${ }^{30}$ KEGG pathway and binding protein analysis consistently indicate the complex role of HSPA5, which participates in a wide variety of signaling and protein-protein interactions. HSPA5, a member of the heat shock protein 70 (HSP70) family, is involved in protein folding and assembly in the endoplasmic reticulum.
Collectively, our results provide insights into the molecular basis underlying the PRMT7-promoted metastasis phenotype of NSCLC cells.

Among 19 candidates, we selected two genes (HSPA5 and $E E F 2$ ) for further studies. According to previous reports, both proteins are shown to be highly involved in the regulation of cancer metastasis. For instance, HSPA5 expression was found to be positively correlated with metastasis in triple-negative breast cancer patients, and downregulation of HSPA5 significantly abolished cancer metastasis in a xenograft tumor model. ${ }^{31}$ In addition, expression of EEF2 in cells increased the capabilities of migration and invasion of cancer cells. ${ }^{32}$ As expected, the interactions between PRMT7 and two candidate proteins (HSPA5 and EEF2) were confirmed with co-immunoprecipitation and Western blot analysis, which suggests the reliability of our experimental protocols. Therefore, it is worth investigating the other 17 identified candidate proteins in future studies (Table 1), which constitutes the focus of our following work.

To assess the effects of both HSPA5 and EEF2 proteins on PRMT7-induced metastasis in NSCLC, we constructed A549 and SPC-A1 cell models bearing both PRMT7 gene overexpression and $H S P A 5 / E E F 2$ gene knockdown. The observation that cell invasion was significantly reduced in both cell lines suggested that these two proteins might serve as essential regulators in the promotion of metastasis
A

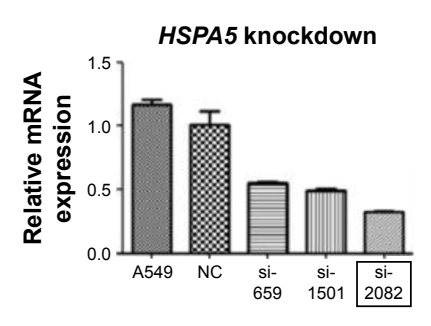

A549

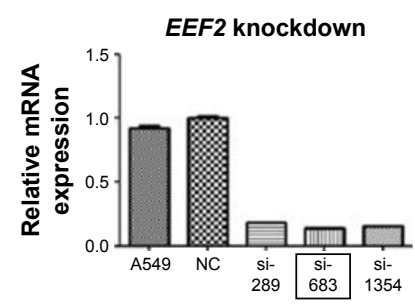

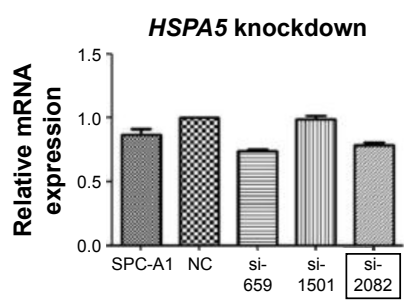

SPC-A1
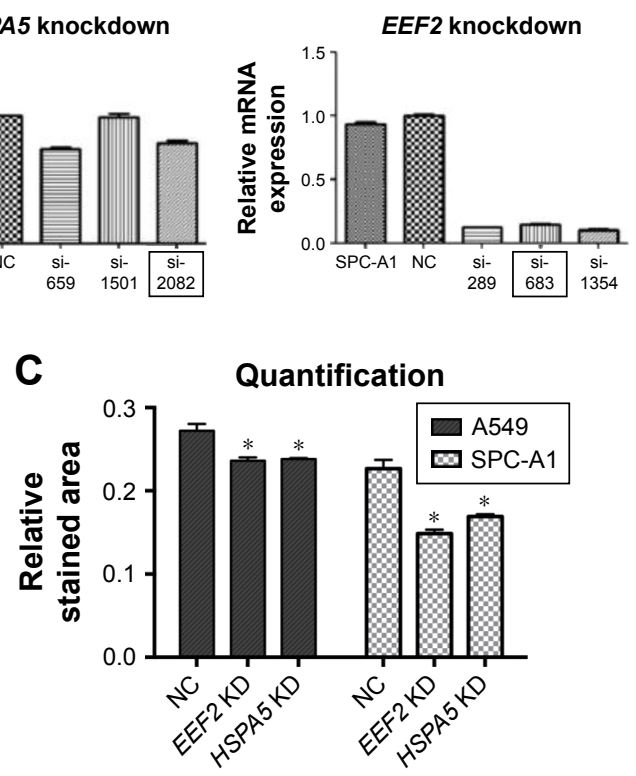

Figure 4 Cell invasion was reduced by gene knockdown of HSPA5 or EEF2.

Notes: (A) mRNA levels of HSPA5 and EEF2 were measured with real-time PCR. (B) The invasion of A549 and SPC-AI cells bearing PRMT7 overexpression was reduced by the gene knockdown of HSPA5 or EEF2. (C) Quantification of cell invasion in A549 and SPC-AI cells. *P $<0.05$, compared to the control group. Abbreviations: PCR, polymerase chain reaction; NC, normal control. 
by PRMT7. In-depth study of the relationship between HSPA 5, EEF2, and PRMT7 will help to clarify the mechanism of PRMT7 in NSCLC metastasis, which also constitutes the main aim of our work.

\section{Acknowledgments}

This work was supported by grants from the Natural Science Foundation of Zhejiang Province (no. LY14H160042) and Wenzhou public welfare science and technology plan (nos. Y20160047 and Y20160049).

\section{Disclosure}

The authors report no conflicts of interest in this work.

\section{References}

1. Siegel R, Ma J, Zou Z, Jemal A. Cancer statistics, 2014. CA Cancer J Clin. 2014;64(1):9-29.

2. Zappa C, Mousa SA. Non-small cell lung cancer: current treatment and future advances. Transl Lung Cancer Res. 2016;5(3):288-300.

3. Sher T, Dy GK, Adjei AA. Small cell lung cancer. Mayo Clin Proc. 2008; 83(3):355-367.

4. Popper HH. Progression and metastasis of lung cancer. Cancer Metastasis Rev. 2016;35(1):75-91.

5. Thomas A, Liu SV, Subramaniam DS, Giaccone G. Refining the treatment of NSCLC according to histological and molecular subtypes. Nat Rev Clin Oncol. 2015;12(9):511-526.

6. Boffa LC, Karn J, Vidali G, Allfrey VG. Distribution of NG, NGdimethylarginine in nuclear protein fractions. Biochem Biophys Res Commun. 1977;74(3):969-976.

7. Yang Y, Bedford MT. Protein arginine methyltransferases and cancer. Nat Rev Cancer. 2013;13(1):37-50.

8. Bedford MT, Clarke SG. Protein arginine methylation in mammals: who, what, and why. Mol Cell. 2009;33(1):1-13.

9. Poulard C, Corbo L, Le Romancer M. Protein arginine methylation demethylation and cancer. Oncotarget. 2016;7(41):67532-67550.

10. Cha B, Jho EH. Protein arginine methyltransferases (PRMTs) as therapeutic targets. Expert Opin Ther Targets. 2012;16(7):651-664.

11. Baldwin RM, Morettin A, Côté J. Role of PRMTs in cancer: Could minor isoforms be leaving a mark? World J Biol Chem. 2014;5(2):115-129.

12. Karkhanis V, Wang L, Tae S, Hu YJ, Imbalzano AN, Sif S. Protein arginine methyltransferase 7 regulates cellular response to DNA damage by methylating promoter histones $\mathrm{H} 2 \mathrm{~A}$ and $\mathrm{H} 4$ of the polymerase $\delta$ catalytic subunit gene, POLD1. J Biol Chem. 2012;287(35):29801-29814.

13. Migliori V, Müller J, Phalke S, et al. Symmetric dimethylation of H3R2 is a newly identified histone mark that supports euchromatin maintenance. Nat Struct Mol Biol. 2012;19(2):136-144.

14. Feng Y, Hadjikyriacou A, Clarke SG. Substrate specificity of human protein arginine methyltransferase 7 (PRMT7): the importance of acidic residues in the double E loop. J Biol Chem. 2014;289(47):32604-32616.

15. Lee SH, Chen TY, Dhar SS, et al. A feedback loop comprising PRMT7 and miR-24-2 interplays with Oct4, Nanog, Klf4 and c-Myc to regulate stemness. Nucleic Acids Res. 2016;44(22):10603-10618.

OncoTargets and Therapy

\section{Publish your work in this journal}

OncoTargets and Therapy is an international, peer-reviewed, open access journal focusing on the pathological basis of all cancers, potential targets for therapy and treatment protocols employed to improve the management of cancer patients. The journal also focuses on the impact of management programs and new therapeutic agents and protocols on
16. Gonsalvez GB, Tian L, Ospina JK, Boisvert FM, Lamond AI, Matera AG. Two distinct arginine methyltransferases are required for biogenesis of Sm-class ribonucleoproteins. J Cell Biol. 2007;178(5):733-740.

17. Thomassen M, Tan Q, Kruse TA. Gene expression meta-analysis identifies chromosomal regions and candidate genes involved in breast cancer metastasis. Breast Cancer Res Treat. 2009;113(2):239-249.

18. Yao R, Jiang H, Ma Y, et al. PRMT7 induces epithelial-to-mesenchymal transition and promotes metastasis in breast cancer. Cancer Res. 2014; 74(19):5656-5667.

19. Martin-Kleiner I. BORIS in human cancers - a review. Eur J Cancer. 2012;48(6):929-935.

20. Jelinic P, Stehle JC, Shaw P. The testis-specific factor CTCFL cooperates with the protein methyltransferase PRMT7 in H19 imprinting control region methylation. PLoS Biol. 2006;4(11):e355.

21. Verbiest V, Montaudon D, Tautu MT, et al. Protein arginine (N)-methyl transferase 7 (PRMT7) as a potential target for the sensitization of tumor cells to camptothecins. FEBS Lett. 2008;582(10):1483-1489.

22. Shin G, Kang TW, Yang S, Baek SJ, Jeong YS, Kim SY. GENT: gene expression database of normal and tumor tissues. Cancer Inform. 2011; 10:149-157.

23. Zufferey R, Nagy D, Mandel RJ, Naldini L, Trono D. Multiply attenuated lentiviral vector achieves efficient gene delivery in vivo. Nat Biotechnol. 1997;15(9):871-875.

24. Asangani IA, Rasheed SA, Nikolova DA, et al. MicroRNA-21 (miR-21) post-transcriptionally downregulates tumor suppressor Pdcd4 and stimulates invasion, intravasation and metastasis in colorectal cancer. Oncogene. 2008;27(15):2128-2136.

25. Kang Y, Zhang R, Suzuki R, et al. Two-dimensional culture of human pancreatic adenocarcinoma cells results in an irreversible transition from epithelial to mesenchymal phenotype. Lab Invest. 2015;95(2):207-222.

26. Li Y, Collins M, An J, et al. Immunoprecipitation and mass spectrometry defines an extensive RBM45 protein-protein interaction network. Brain Res. 2016;1647:79-93.

27. Baldwin RM, Haghandish N, Daneshmand M, et al. Protein arginine methyltransferase 7 promotes breast cancer cell invasion through the induction of MMP9 expression. Oncotarget. 2015;6(5):3013-3032.

28. Tiscornia G, Singer O, Ikawa M, Verma IM. A general method for gene knockdown in mice by using lentiviral vectors expressing small interfering RNA. Proc Natl Acad Sci U S A. 2003;100(4):1844-1848.

29. Jain K, Jin CY, Clarke SG. Epigenetic control via allosteric regulation of mammalian protein arginine methyltransferases. Proc Natl Acad Sci US A. 2017;114(38):10101-10106.

30. Palmer TD, Ashby WJ, Lewis JD, Zijlstra A. Targeting tumor cell motility to prevent metastasis. Adv Drug Deliv Rev. 2011;63(8):568-581.

31. Chen HA, Chang YW, Tseng CF, et al. E1A-mediated inhibition of HSPA 5 suppresses cell migration and invasion in triple-negative breast cancer. Ann Surg Oncol. 2015;22(3):889-898.

32. Song Y, Sun B, Hao L, et al. Elevated eukaryotic elongation factor 2 expression is involved in proliferation and invasion of lung squamous cell carcinoma. Oncotarget. 2016;7(36):58470-58482.

33. Kim JH, Yoo BC, Yang WS, Kim E, Hong S, Cho JY. The Role of Protein Arginine Methyltransferases in Inflammatory Responses. Mediators Inflamm. 2016;2016:4028353.

34. Morales Y, Cáceres T, May K, Hevel JM. Biochemistry and regulation of the protein arginine methyltransferases (PRMTs). Arch Biochem Biophys. 2016;590:138-152.

patient perspectives such as quality of life, adherence and satisfaction The manuscript management system is completely online and includes a very quick and fair peer-review system, which is all easy to use. Visit http://www.dovepress.com/testimonials.php to read real quotes from published authors. 